Supplementary Material to:

\title{
Statitical tests against systematic errors in data sets based on the equality of residual means and variances from control samples: Theory and Applications
}

\author{
Julian Henn $^{* a}$ and Kathrin Meindl ${ }^{\mathrm{b}}$ \\ Emil-Warburg Weg 6, 95447 Bayreuth, Germany and Instituto de Biologia Molecular de \\ Barcelona (IBMB-CSIC), Barcelona Science Park, Baldiri Reixach, 08028 Barcelona, Spain. \\ E-mail: Julian.Henn@uni-bayreuth.de
}

The supplementary material contains 9 diagnostic plots (with to some extent redundant information) for each of the data sets $5,6,7,12,13,24$ and additionally for data set 5 with 2 omitted outliers, data set 24 with cut off, data set 24 with manipulated s.u.'s, and data set 24 with 5 intentionally flawed reflections. A Figure is included that shows plots of residuals vs resolution and of observed versus calculates intensities for artificial data known to be free of systematic errors and contaminated with systematic errors due to inappropriate standard uncertainties in one case and due to 5 intentionally flawed intensity observations in the other case. 


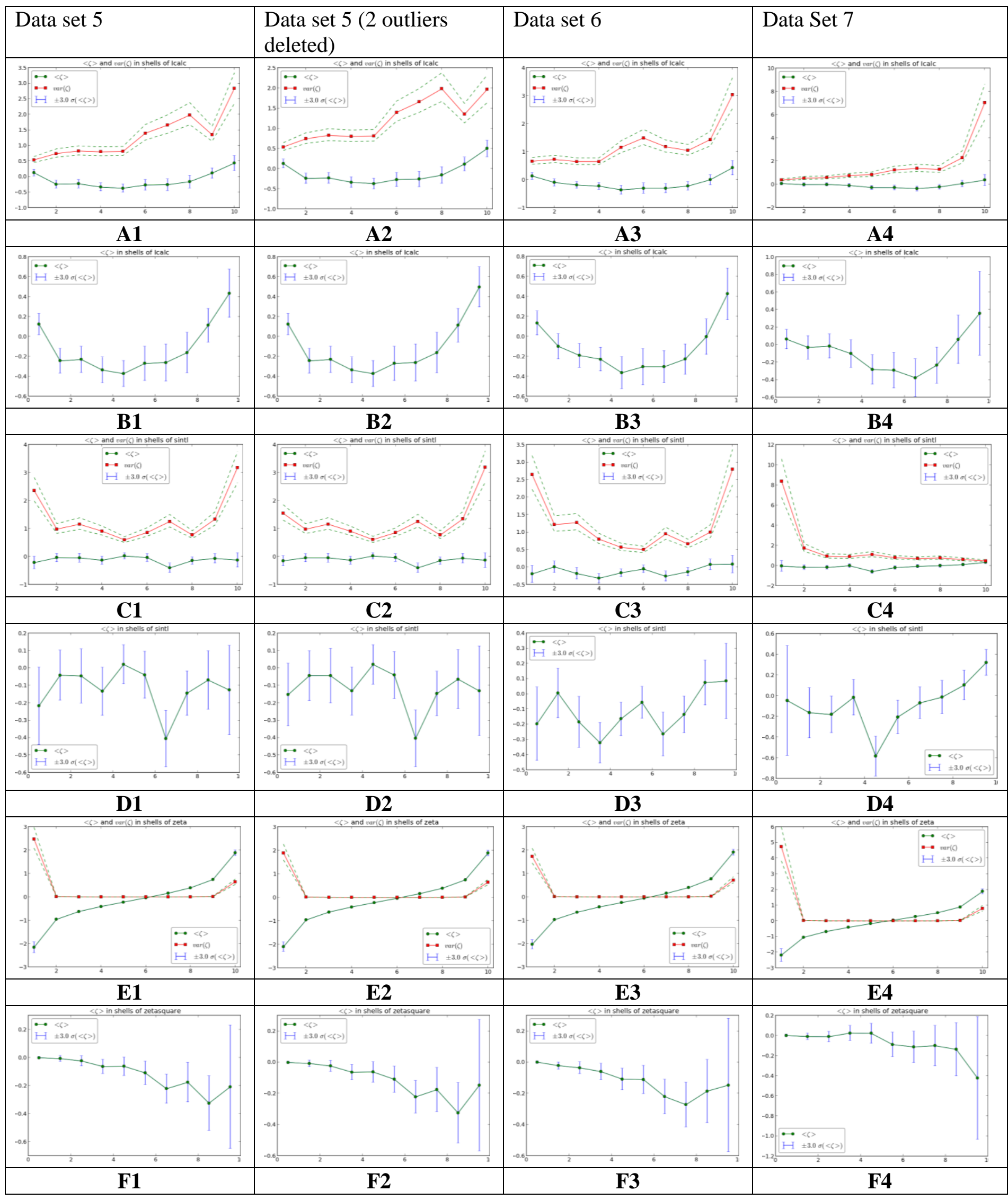




\begin{tabular}{l} 
K1 \\
\hline $\begin{array}{l}\text { Fig. 1: Residual mean values and residual's mean variances sorted by increasing intensity, (A), residual mean } \\
\text { values sorted by increasing intensity,(B), Residual mean values and residual's mean variances sorted by } \\
\text { increasing resolution, (C), residual mean values sorted by increasing resoution,(D), residual mean values and } \\
\text { residual's mean variances sorted by residuals, (E), residual mean values sorted by squared residuals, (F), } \\
\text { cumulative rare events sorted by increasing resolution, (G), and by increasing intensity, (H), and normal } \\
\text { probability plots, (K) for data sets 5-7. }\end{array}$ \\
\hline
\end{tabular}




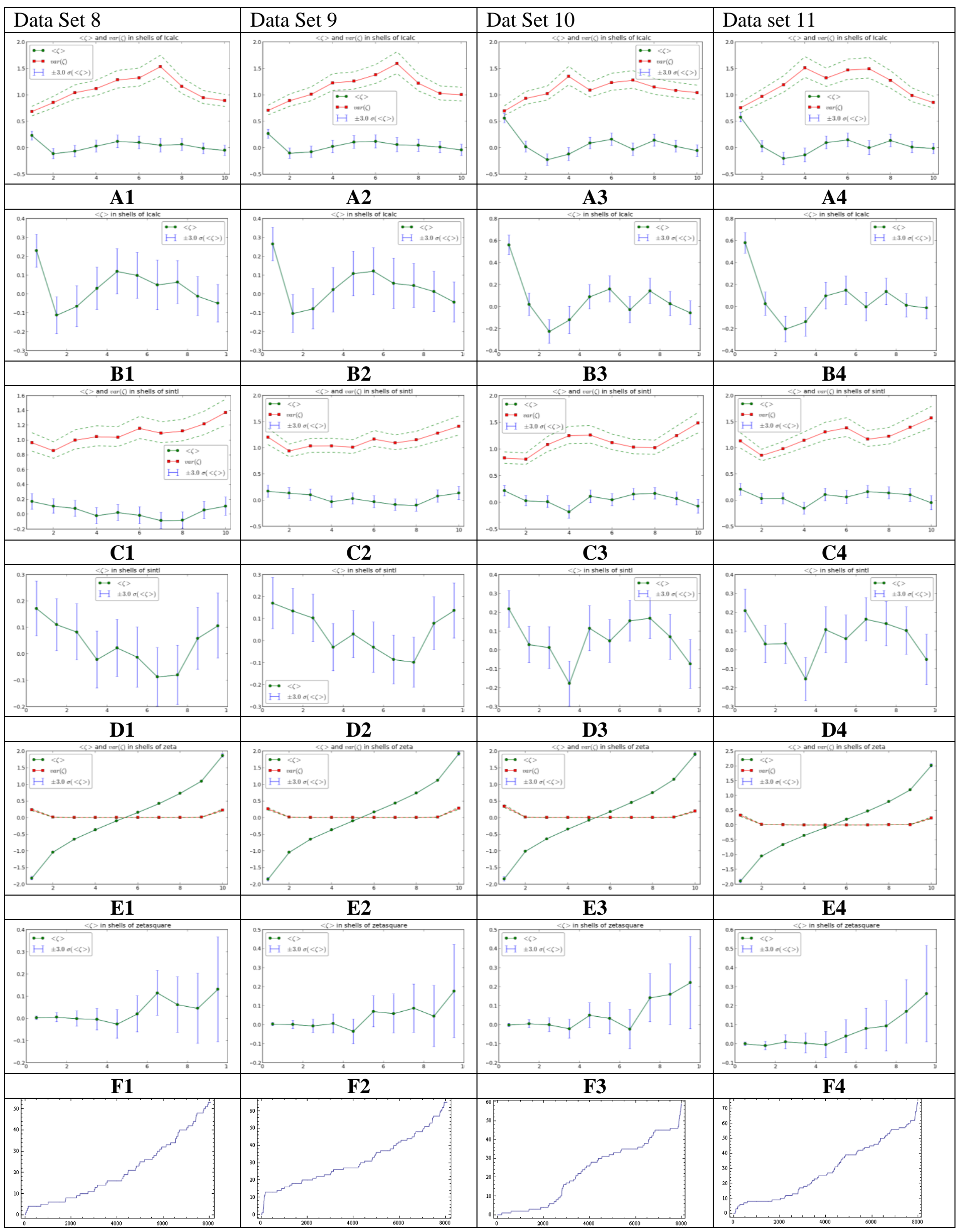




K1
Fig. 2: Residual mean values and residual's mean variances sorted by increasing intensity, (A), residual mean
values sorted by increasing intensity,(B), Residual mean values and residual's mean variances sorted by
increasing resolution, (C), residual mean values sorted by increasing resoution, (D), residual mean values and
residual's mean variances sorted by residuals, (E), residual mean values sorted by squared residuals, (F),
cumulative rare events sorted by increasing resolution, (G), and by increasing intensity, (H), and normal
probability plots, (K) for data sets 8-11.




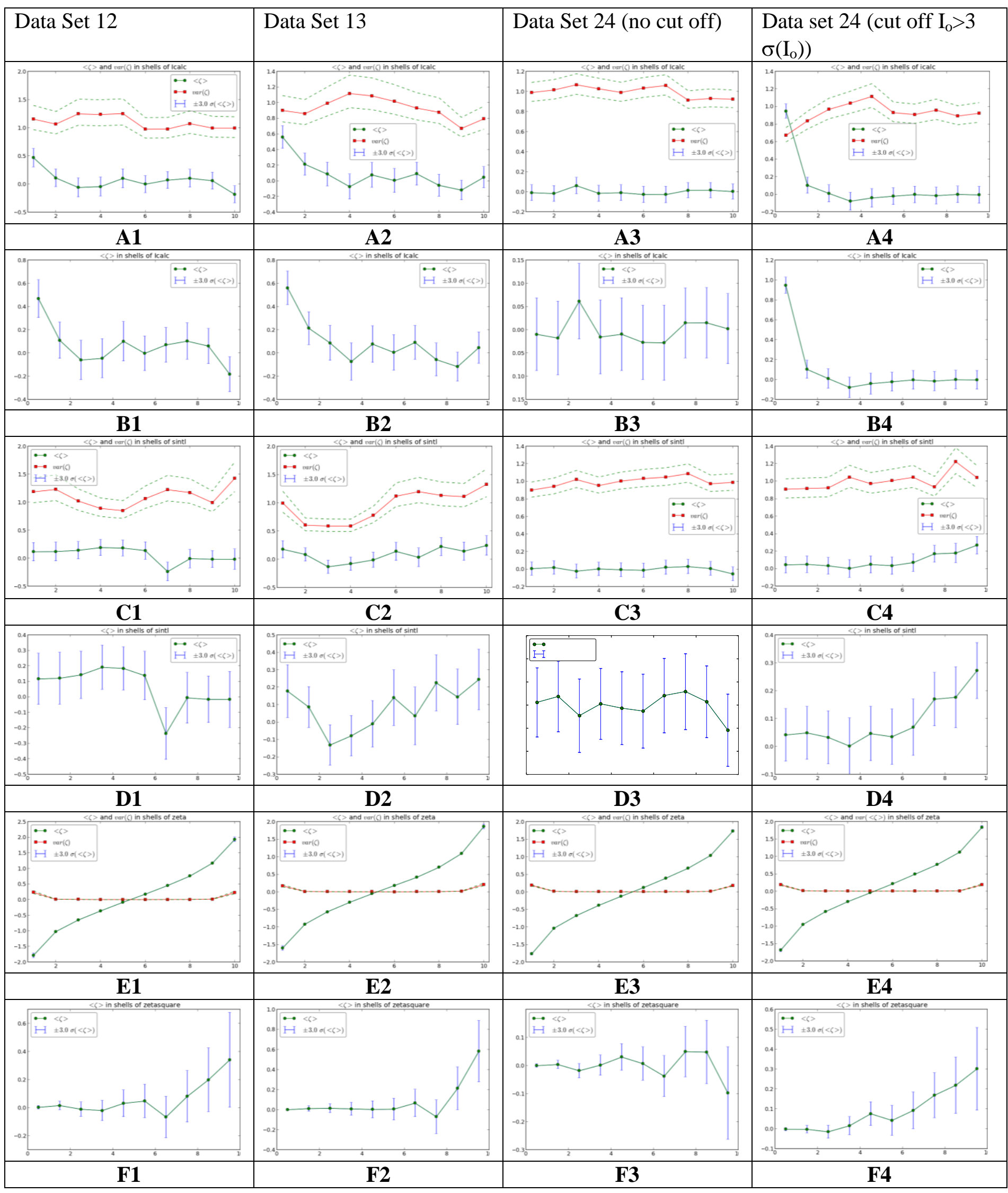




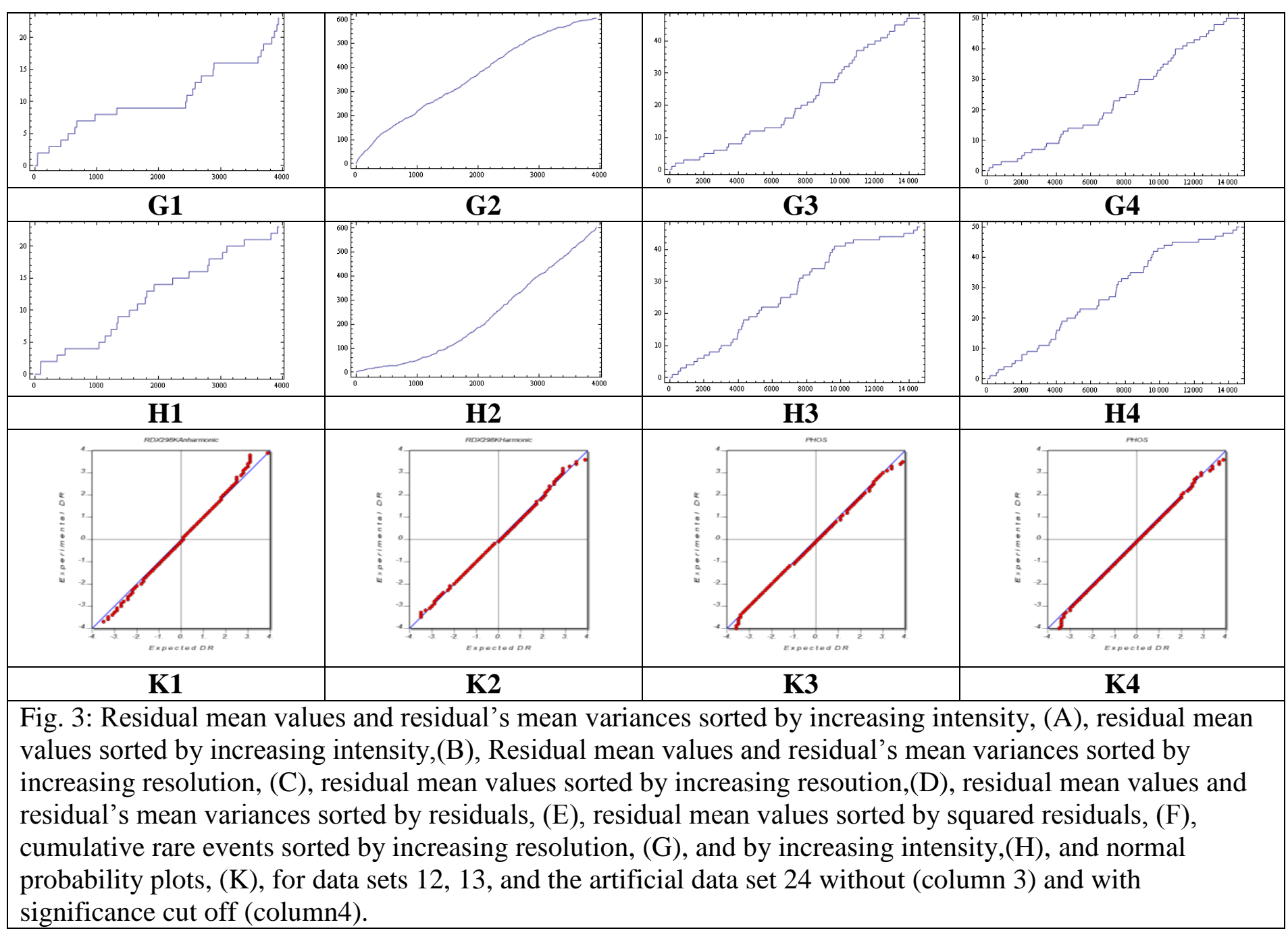




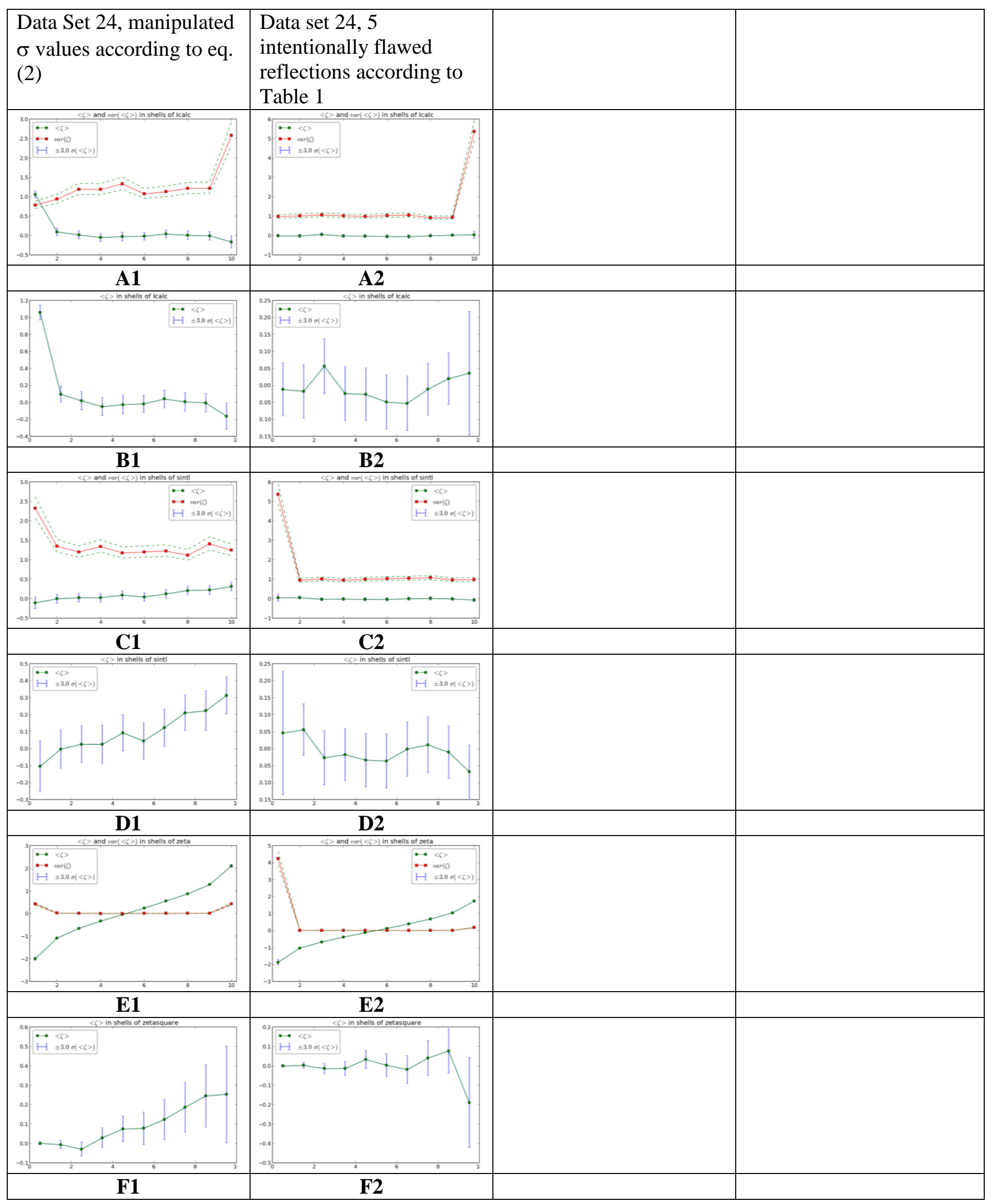




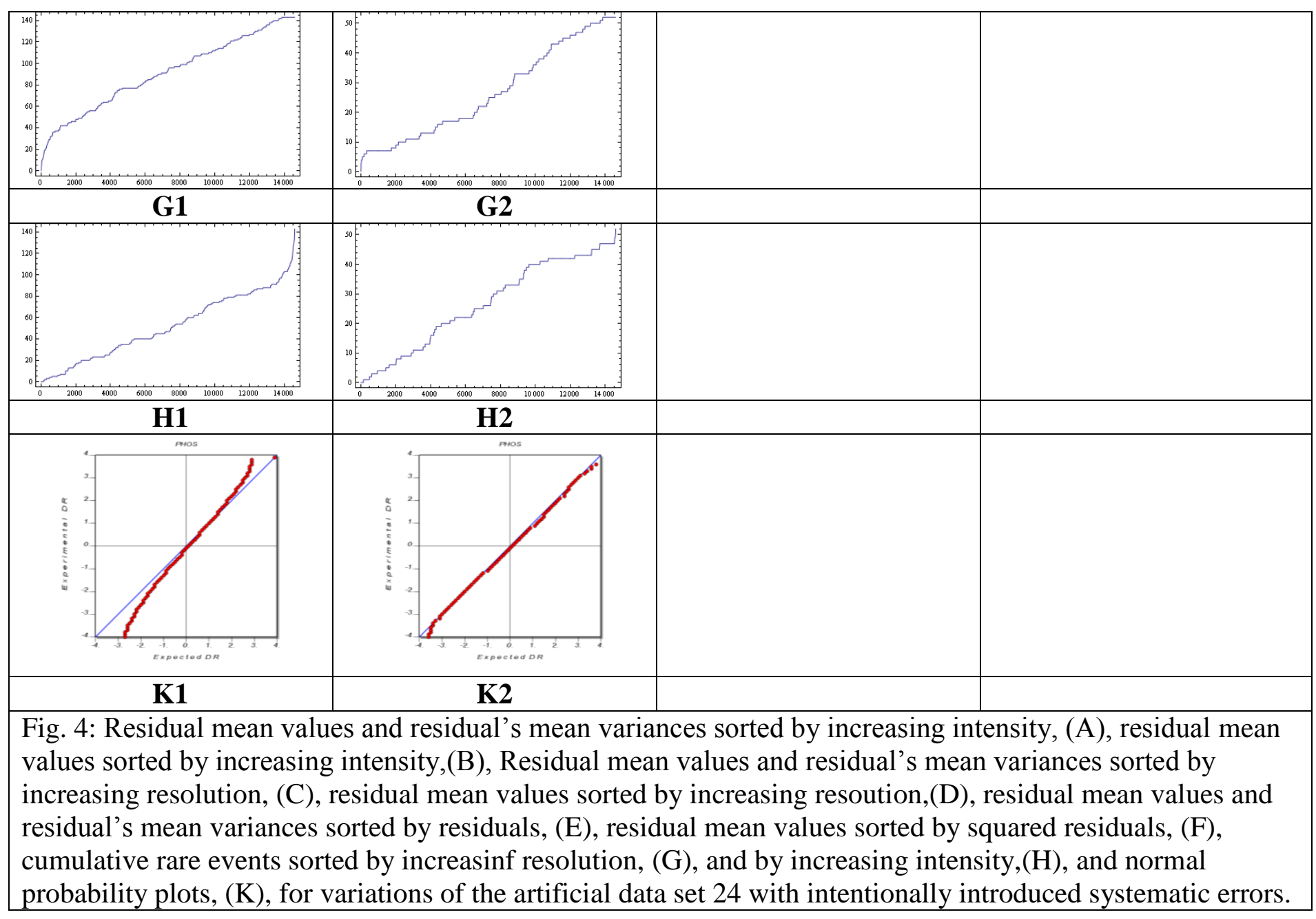




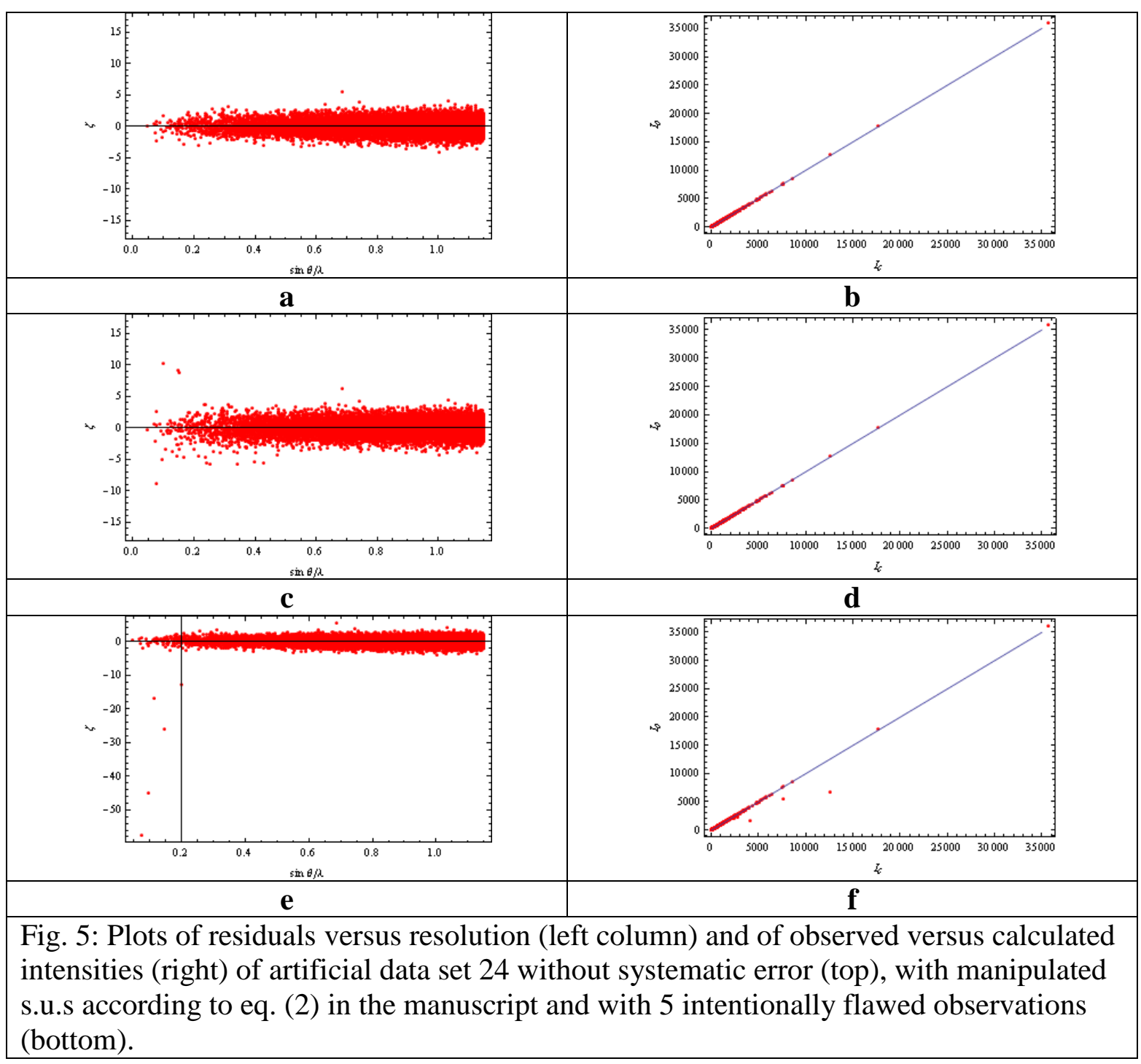

